Journal of the Mathematical Society of Japan Vol. 3, No. 1, May, 1951.

\title{
Multiple Wiener Integral
}

Kiyosi ITô

The notion of multiple Wiener integral was introduced first by $\mathrm{N}$. Wiener ${ }^{1)}$ who termed it polynomial chaos. Our definition in the present paper is obtained by a slight modification of Wiener's one, and seems to be more convenient in the point that our integrals of different degrees are orthogonal to each other while Wiener's polynomial cháos has not such a property.

In $\S 1$ we shall define a normal random measure as a generalization of a brownian motion process. In $\S 2$ we shall define multiple Wiener integral and show its fundamental property. In $\S 3$ we shall establish å close relation between our integrals and Hermite polynomials. By making use of this relation we shall give, in $\S 4$, an orthogonal expansion of any $L_{2}$-functional of the normal random measure, which proves to be coincident with the expansion given by $\mathrm{S}$. Kakutani ${ }^{2)}$ for the purpose of the spectral resolution of the shift operator in the $L_{2}$ over the brownian motion process. In $\$ 5$ we shall treat the case of a brownian motion process, and- in this case we shall show that we can define the multiple Wiener integral by the iteration of stochastic integrals. ${ }^{3)}$

\section{$\S 1$. Normal random measure}

A system of real random veriables $\xi_{\alpha}(\omega), \alpha \epsilon A, \omega$ being a probability parameter, is called normal when the joint destibution of $\hat{\xi}_{\alpha_{1}}, \ldots, \xi_{\alpha_{n}} ; \alpha_{1}, \ldots$, $u_{n} \in A$, is always a multivariate Gaussian distribution (including degenerate cases) with the mean vector $(0, \cdots, 0)$.

By making use of Kolmogoroff's theorem ${ }^{4)}$ of introducing a probability distribution in $R^{A}$, we can easily prove the following

1) N. Wiener: The homogeneous chaos, Amer. Journ. Math. Vol. $\boldsymbol{L}$, No. 4, 1938.

2) S. Kakntani : Determination of the spectrum of the flow of Brownian motion, Proc. Nat. Acad. Sci., U.S.A., 36 (1950), 319-323.

3) K. Itô : Stochastic integral, Proc. Imp. Acad. Tokyo, Vol. $\boldsymbol{X} \boldsymbol{X}$, No. 8, 1944.

4) A. Kolmogoroff : Grundbegriffe der Wahrscheinlichkeitsrechnung, Berlin, 1933. The consistency-condition is well satisfied by virtue of the property of multivariate Gaussian distribution. 
Theorem 1. 1 If $v_{\alpha \beta} ; \alpha, \beta \in A$, satisfies the following two conditions: symmetric : $v_{\alpha \beta}=v_{\beta \alpha}$;

positive-definite : $\sum x_{i} \bar{x}_{j} v_{\alpha_{i} \alpha_{i}} \geqq 0$ (for any $\alpha_{1}, \cdots, \alpha_{n} \in A$ and for any complex numbers $\left.x_{1}, x_{2}, \cdots, x_{n}\right)$, then there exists a normal random system $\xi_{\alpha}, a \in A$, zulich satisfies

$$
v_{\alpha \beta}=\left(\xi\left(\xi_{\alpha} \xi_{\beta}\right)=\int \xi_{\alpha}(\omega) \xi_{\beta}(\omega) d \omega .\right.
$$

Definition. Let $(T, \boldsymbol{B}, m)$ be a measure space. We denote by $\boldsymbol{B}^{*}$ the system $\{E ; E \in \boldsymbol{B}, m(E)<\infty\}$. A normal system $\beta(E, \omega), E \in \boldsymbol{B}^{*}$, is called a normal random measure on $(T, \boldsymbol{B}, m)$, if

$$
\mathfrak{E}\left(\beta(E) \beta\left(E^{\prime}\right)\right)=m\left(E \cap E^{\prime}\right) \text { for any } E, E^{\prime} \in \boldsymbol{B}^{*} \text {. }
$$

Remark: Since we have $m\left(E \cap E^{\prime}\right)=m\left(E^{\prime} \cap E\right)$ and $\sum x_{i} \bar{x}_{j} m\left(E_{i} \cap E_{j}\right)$ $=\int\left|\sum_{i} x_{i} C_{i}(t)\right|^{2}, m(d t) \geqq 0, C_{i}(t)$ being the characteristic function of the set $E_{i}$, we can see, by Theorem 1.1 , the existence of a normal random measure on any measure space $(T, \boldsymbol{B}, m)$.

The following theerem, which can be easily shown, justifies the name of normal random " measure."

Theorem. 1. 2 Let $\beta(E)$ be a normal random measure on $(T, \boldsymbol{B}, m)$. If $E_{1}, E_{2}, \cdots$ are disjoint, then $\beta\left(E_{1}\right), \beta\left(E_{2}\right), \cdots$ are independent. Furthermore if $E=E_{1}+E_{2}+\cdots \in \boldsymbol{B}^{*}$, then $\beta(E)=\sum_{n} \beta\left(E_{n}\right)$ (in mean convergence).

Remark. Since $\beta\left(E_{1}\right), \beta\left(E_{2}\right), \cdots$, are independent, then the mean convergence of $\Sigma \beta\left(E_{n}\right)$ implies the almost certain convergence by virtue of Levy's theorem. ${ }^{\text {) }}$

Hereafter we set the following restriction on the measure $m$.

Continuity. For any $E \in \boldsymbol{B}^{*}$ and $\varepsilon>0$ there exists a decomposition of $E$ :

$$
E=\sum_{i=1}^{n} E_{i}
$$

such that

$$
m\left(E_{i}\right)<\varepsilon, i=1,2, \cdots, n \text {. }
$$

5) P. Lévy: Théorie de l'addition des variariables aléatoires, Paris, 1937. 


\section{§ 2. Definition of multiple Wiener integral}

By $L^{2}\left(T^{p}\right)$ we denote the totality of square-summable complex-valued functions defined on the product measure space $(T, \boldsymbol{B}, m)^{p}$. An elementary function ${ }^{6)} f\left(t_{1}, \cdots, t_{p}\right)$ is called special if $f\left(t_{1}, \cdots, t_{p}\right)$ vanishes except for the case that $t_{1}, \cdots, t_{p}$ are all different. We shall denote by $S_{p}$ the totality of special elementary functions.

Theorem 2. 1. $S_{p}$ is a linear manifold dense in $L^{2}\left(T^{p}\right)$.

Proof. It suffices to show that the characteristic function $c\left(t_{1}, \cdots, t_{p}\right)$ of any set $E$ of the form :

$$
E=E_{1} \times E_{2} \times \cdots \times E_{p}\left(E_{i} \in \boldsymbol{B}^{*}, i=1,2, \cdots, p\right)
$$

can be approximated (in the $L_{2}$-norm) by a special elementary function.

For any $\varepsilon>0$ we can determine, by the continuity condition, a setsystem $\boldsymbol{H}=\left\{F_{1}, \cdots, F_{n}\right\} \in \boldsymbol{B}^{*}$ which satisfies

F. 1. $F_{1}, F_{2}, \cdots, F_{n}$ are disjoint,

F. 2. $m\left(F_{i}\right)<\varepsilon_{1} \equiv \varepsilon /\left(\begin{array}{l}p \\ 2\end{array}\right) \cdot\left(\sum m\left(E_{i}\right)\right)^{p-1},\left(\begin{array}{l}p \\ 2\end{array}\right)=\frac{p(p-1)}{2 \cdot 1}$,

F. 3. each $E_{i}$ is expressible as the sum of a subsystem of $\boldsymbol{F}$.

Then $c\left(t_{1}, \cdots, t_{p}\right)$ is expressible in the form:

$$
c\left(t_{1}, \cdots, t_{p}\right)=\sum \varepsilon_{i_{1}} \ldots i_{p} c_{i_{1}}\left(t_{1}\right) \ldots c_{i_{1}}\left(t_{p}\right)
$$

where $\varepsilon_{i_{1} \cdots i_{p}}=0$ or 1 and $c_{i}(t)$ is the characteirstic function of $F_{i}, i=1$, $2, \ldots, n$. We devide $\Sigma$ into two parts: $\Sigma^{\prime}$ and $\Sigma^{\prime \prime}: \Sigma^{\prime}$ corresponds to the indices $\left\{i_{1}, \ldots, i_{p}\right\}$ which are all different, while $\Sigma^{\prime \prime}$ corresponds to the others.

We put

$$
f\left(t_{1}, \ldots, t_{p}\right)=\Sigma^{\prime} \varepsilon_{i_{1} \ldots i_{p} c_{i}} c_{i_{1}}\left(t_{1}\right) \ldots c_{i_{p}}\left(t_{p}\right) .
$$

Then $f \in S_{p}$ and

$$
\begin{aligned}
\|c-f\|^{2} & =\int \cdots \int\left|c\left(t_{1}, \ldots, t_{p}\right)-f\left(t_{1}, \cdots, t_{p}\right)\right|^{2} m\left(d t_{1}\right) \ldots m\left(d t_{p}\right) \\
& =\Sigma^{\prime \prime} \varepsilon_{i_{1} \cdots i_{p}} m\left(F_{i_{1}}\right) \ldots m\left(F_{i_{p}}\right)
\end{aligned}
$$

6) An elementary function of $\left(t_{1}, \ldots, t_{p}\right)$ is defined as a linear combination of the characteristic functions of the sets of the form $E_{1} \times \ldots \times E_{p}, E_{i} \in \boldsymbol{B}^{*}, i=12, \ldots, n$. 


$$
\begin{aligned}
& \leqq\left(\begin{array}{c}
p \\
2
\end{array}\right) \sum m\left(F_{i}\right)^{2}\left(\sum m\left(F_{i}\right)\right)^{p-2} \\
& \leqq\left(\begin{array}{c}
p \\
2
\end{array}\right) \varepsilon_{1}\left(\sum m\left(F_{i}\right)\right)^{p-1}=\left(\frac{p}{2}\right) \varepsilon_{1}\left(\sum m\left(E_{i}\right)\right)^{p-1}<\varepsilon .
\end{aligned}
$$

Now we shall define the multiple wiener integral of $f \in L^{2}\left(T^{p}\right)$, which we denote by

$$
I_{p}(f) \text { or } \int \ldots \int f\left(t_{1}, \ldots, t_{p}\right) d \beta\left(t_{1}\right) \ldots d \beta\left(t_{p}\right)
$$

Let $f$ be a special elementary function. Then $f$ can be expressible as follows :

$$
\begin{aligned}
f\left(t_{1}, \ldots, t_{p}\right) & =a_{i_{1} \ldots i_{p}} \text { for }\left(t_{1}, \ldots, t_{p}\right) \in T_{t_{1}} \times \ldots \times T_{i_{p}}, \\
& =0 \quad \text { elsewhere, }
\end{aligned}
$$

where $T_{1}, T_{2}, \ldots, T_{n}$ are disjoint and $m\left(T_{i}\right)<\infty, i=1,2, \ldots, n$, and $a_{i_{1} \ldots i_{p}}=0$ if any two of $i_{1}, \ldots, i_{p}$ are equal. We define $I_{p}(f)$ for such $f$ by

$$
I_{p}(f)=\sum a_{i_{1} \ldots i_{p}} \beta\left(T_{i_{1}}\right) \ldots \beta\left(T_{i_{p}}\right) .
$$

Then we obtain

$$
\begin{aligned}
& I_{p}(a f+b g)=a I_{p}(f)+b I_{p}(g) \\
& I_{p}(f)=I_{p}(\tilde{f}),
\end{aligned}
$$

where

$$
\tilde{f}\left(t_{1}, \ldots, t_{p}\right)=\frac{1}{\underline{p}} \sum_{(\pi)} f\left(t_{\pi_{1}}, \ldots, t_{\pi_{p}}\right),(\pi)=\left(\pi_{1}, \ldots, \pi_{p}\right) \text { running over all per- }
$$
mutations of $(1,2, \ldots, p)(\mid \underline{p}=1 \cdot 2 \cdot \ldots \cdot p)$.

$$
\left(I_{p}(f), I_{p}(g)\right)=\underline{p}(\tilde{f}, \tilde{g}),
$$

where $\left(I_{p}(f), I_{p}(g)\right) \equiv \mathbb{E}\left(I_{p}(f) \overline{I_{p}(g)}\right) \equiv \int I_{p}(f) \cdot \overline{I_{p}(g)} d \omega$, and

$$
\begin{aligned}
(\tilde{f}, \tilde{g}) \equiv \int \ldots \int \tilde{f}\left(t_{1}, \ldots, t_{p}\right) \overline{\widetilde{s}\left(t_{1}, \ldots, t_{p}\right)} m\left(d t_{1}\right) \ldots m\left(d t_{p}\right) . \\
\left(I_{p}(f), I_{p}(g)\right)=0, \text { if } p \neq q .
\end{aligned}
$$


(I.1) is clear. In order to show (I.2) and (I.3) we may assume that $f$ and $g$ are expressible as follows:

$$
\begin{array}{r}
f\left(\imath_{1}, \ldots, t_{p}\right)=a_{i_{1}} \cdots t_{p}, g\left(t_{1}, \ldots, t_{p}\right)=b_{i_{1}} \ldots t_{p} \\
\text { for }\left(t_{1}, \ldots, t_{p}\right) \in T_{i_{1}} \times \cdots \times T_{i_{p}}
\end{array}
$$

and

$$
f\left(t_{1}, \ldots, t_{p}\right)=0, g\left(t_{1}, \ldots, t_{p}\right)=0 \quad \text { elsewhere. }
$$

Then we have

$$
\begin{aligned}
I_{p}(f) & =\sum_{i_{1}<\cdots<i_{p}}\left(\sum_{(j) \sim(t)} a_{j_{1} \cdots j_{p}}\right) \beta\left(T_{i_{1}}\right) \ldots \beta\left(T_{i_{p}}\right)^{7)} \\
& =\underline{\mid p} \sum_{i_{1}<\cdots<i_{p}}\left(\frac{1}{\underline{p}} \sum_{(j) \sim(i)} a_{j_{1} \cdots j_{p}}\right) \beta\left(T_{i_{1}}\right) \ldots \beta\left(T_{t_{p}}\right) \\
& =\sum_{i_{1}}, \cdots, i_{p} \\
& =I(\underline{\underline{f}}),\left(\underline{\underline{p}} \sum_{(j) \sim(\delta)} a_{j_{1} \cdots \cdot j_{p}}\right) \beta\left(T_{i_{1}}\right) \ldots \beta\left(T_{t_{p}}\right)
\end{aligned}
$$

which proves (I.2).

$$
\begin{aligned}
& \left(I_{p}(f), I_{p}(g)\right)=\left(\sum_{i_{1}<\cdots<i_{p}}\left(\sum_{(j) \sim(i)} a_{j_{1} \cdots j_{p}}\right) \beta\left(T_{i_{1}}\right) \ldots \beta\left(T_{i_{p}}\right),\right. \\
& \left.\sum_{i_{1}<\cdots<i_{p}}\left(\sum_{(j) \sim(i)} b_{j_{1} \cdots g_{p}}\right) \beta\left(T_{i_{1}}\right) \ldots \beta\left(T_{i_{p}}\right)\right) \\
& =\sum_{i_{1}<\cdots<i_{p}}\left(\sum_{(j) \sim(i)} a_{j_{1} \cdots j_{p}}\right) \cdot\left(\sum_{(j) \sim(i)} \bar{b}_{j_{1} \cdots j_{p}}\right) m\left(T_{i_{1}}\right) \ldots m\left(T_{i_{p}}\right) \\
& =\frac{1}{\underline{\underline{p}}} \sum_{i_{1} \cdots i_{p}}\left(\sum_{(j) \sim(i)} a_{j_{1} \cdots j_{p}}\right)\left(\sum_{(j) \sim(i)} \bar{b}_{j_{1} \cdots j_{p}}\right) m\left(T_{i_{1}}\right) \ldots m\left(T_{i_{p}}\right) \\
& =\underline{p} \sum_{i_{i} \cdots i_{p}}\left(\frac{1}{\underline{p}} \sum_{(j) \sim(i)} a_{j_{1} \cdots j_{p}}\right)\left(\underline{\underline{1}} \sum_{(j) \sim(i)} b_{j_{1} \cdots j_{p}}\right) m\left(T_{i_{1}}\right) \cdots m\left(T_{i_{p}}\right) \\
& =\mid p \int \ldots \int \tilde{f\left(t_{1}, \ldots, t_{p}\right) \cdot \overline{\tilde{g}}\left(t_{1}, \ldots, t_{p}\right)} m\left(d t_{1}\right) \ldots m\left(d t_{p}\right) \\
& =\underline{p}(\tilde{f}, \tilde{s}) \text {. }
\end{aligned}
$$

7) $(j) \sim(i)$ means that $(j) \equiv\left(j_{1}, \ldots j_{q}\right)$ is a permutation of $(i) \equiv\left(i_{1}, \ldots, i_{p}\right)$. 
Thus (I.3) is proved.

By the similar computations we can prove (I.4).

By putting $f=g$ in $(I .3)$, we obtain

$$
\left\|I_{p}(f)\right\|^{2}=\underline{\mid p}\|\tilde{f}\|^{2} \leqq \underline{\mid p}\|f\|^{2}
$$

the last inequality being obtained by virtue of Schwarz' inequality.

Therefore $I_{p}$ can be considered as a bounded linear operator from $S_{p}$ into $L_{2}(\omega)$, and so it can be extended to an operator from the closure of $S_{p}\left(=L^{2}\left(T^{p}\right)\right.$ by Theorem 2.1) into $L_{2}(\omega)$ which satisfies also $(I .1),(I .2)$, $(I .3),(I .4)$ and $\left(I .3^{\prime}\right)$.

For the later use we denote by $L_{0}^{2}$ the totality of complex numbers and we define as $I_{0}(c)=c$. Thus $(I .1),(I .2),(I .3),(I .4)$ and $\left(I .3^{\prime}\right)$ are true for $p, q=0,1,2, \ldots$

\section{§ 3. Relation between multiple Wiener integrals and Hermite polynomials.}

Theorem 3.1. Let $\varphi_{1}(t), \varphi_{2}(t), \ldots, \varphi_{n}(t)$ be an orthogonal system of real-salued functions in $L^{2}(T)$ and $H_{p}(x)$ bc the Hirmitc polynomial of digree p. Then we have

$$
\begin{aligned}
\int \ldots \int & \psi_{1}\left(t_{1}\right) \ldots \phi_{1}\left(t_{p_{1}}\right) \cdot \varphi_{2}\left(t_{p_{1}+1}\right) \ldots \varphi_{2}\left(t_{p_{1}+p_{2}}\right) \ldots \\
& \times \varphi_{n}\left(t_{p_{1}+\cdots+p_{n-1}+1}\right) \ldots \varphi_{n}\left(t_{p_{1}+\cdots+p_{n}}\right) d \beta\left(t_{1}\right) \ldots d \beta\left(t_{p_{1}+\cdots+p_{n}}\right) \\
& =\prod_{\nu=1}^{n} \frac{H_{p_{\nu}}\left(\frac{1}{\sqrt{2}} \int \varphi_{\nu}(t) d \beta(t)\right)}{\sqrt{2}^{p_{\nu}}} .
\end{aligned}
$$

For the proof of this theorem we prepare the following

Theorem 2.2. I, If $\varphi\left(t_{1}, \ldots, t_{p}\right) \in L^{2}\left(T^{p}\right)$ and $\phi(t) \in L^{2}(T)$, then

$$
\begin{gathered}
\int \ldots\left[\left|\varphi\left(t_{1}, \ldots, t_{p}\right) \psi\left(t_{k}\right)\right| m\left(d t_{k}\right)\right]^{2} m\left(d t_{1}\right) \ldots m\left(d t_{k-1}\right) m\left(d t_{k-1}\right) \ldots m\left(d t_{p}\right) \\
\leqq\|\psi\|^{2} \cdot\|\varphi\|^{2}<\infty .
\end{gathered}
$$

Therefore

II. $\varphi \underset{(k)}{\times} \phi\left(t_{1} \ldots t_{k-1} t_{k+1} \ldots t_{p}\right) \equiv \int \varphi\left(t_{1}, \ldots, t_{p}\right) \phi\left(t_{k}\right) m\left(d t_{k}\right)$ 
is a square-summable function of $t_{1}, \cdots, t_{k-1}, t_{k+1}, \cdots, t_{p}$, and it holds

$$
\|\varphi \underset{(k)}{\times} \phi\| \leqq\|\varphi\| \cdot\|\phi\| .
$$

III. We have

$$
I_{p+1}(\varphi \psi)=I_{p}(\varphi) \cdot I_{1}(\phi)-\sum_{k=1}^{p} I_{p-1}(\underset{(k)}{\times} \psi)
$$

Proof. (3.2) is clear by virtue of Schwarz' inequality and (3.3) is also true by the definition of the norm $\|$. $\|$ in $L_{2}$. For the proof of (3.4) we consider firstly the case when $\varphi$ and $\phi$ are special elementary functions. Then we may express $\varphi$ and $\phi$ in the form

$$
\begin{aligned}
\varphi\left(t_{1}, \ldots, t_{p}\right) & =a_{i_{1} \cdots i_{p}} \text { for }\left(t_{1}, \ldots, t_{p}\right) \in T_{i_{1}} \times \ldots \times T_{i_{p}}, \\
& =0 \quad \text { elsewhere, } \\
\varphi(t) & =b_{i} \quad \text { for } \quad t_{i} \in T_{i} \\
& =0 \quad \text { elsewhere, }
\end{aligned}
$$

where $T_{1}, T_{2}, \ldots, T_{N}$ are disjoint and $m\left(T_{i}\right)<\infty, i=1,2, \ldots, N$ and $a_{i_{1}} \cdots i_{p}$ $=0$ if any two of $i, \ldots, i_{p}$ are equal.

Put $S=T_{1}+\ldots+T_{N}, A=\dot{\max }\left|a_{i}\right|$, and $B=\max \left|b_{i}\right|$. Then

$$
m(S), A, B<\infty
$$

On account of the continuity-condition of $m$ we may assume that

$$
m\left(T_{i}\right)<\varepsilon, i=1,2, \ldots, N,
$$

for any asigned $\varepsilon>0$, by subdividing each $T_{i}$, if necessary. $S, A$ and $B$ remain invariant by this subdivision.

Now we define a special elementary function $\chi_{\varepsilon}$ by

$$
\begin{gathered}
\chi_{\varepsilon}\left(t_{1}, \ldots, t_{p}, t\right)=a_{i_{1} \ldots i_{p}} b_{i}, \quad \text { if }\left(t_{1}, \ldots, t_{p}, t\right) \in T_{i_{1}} \times \ldots \times T_{i_{p}} \times T_{i}, \\
\text { and } i \neq i_{1}, \ldots, i_{p} . \\
=0, \quad \text { if otherwise. }
\end{gathered}
$$

Then we have

$$
I_{p}(\varphi) \cdot I_{1}(\psi)=\sum a_{i_{1} \ldots i_{p}} \beta\left(T_{i_{1}}\right) \ldots \beta\left(T_{i_{p}}\right) \sum b_{i} \beta\left(T_{i}\right)
$$




$$
\begin{aligned}
& =\sum_{i \neq i_{1}, \cdots, i_{p}} a_{i_{1} \cdots i_{p}} b_{i} \beta\left(T_{i_{1}}\right) \ldots \beta\left(T_{i_{p}}\right) \beta\left(T_{i}\right) \\
& +\sum_{k=1}^{p} \sum a_{t_{1} \ldots t_{p}} b_{t_{k}} \beta\left(T_{i_{1}}\right) \ldots \beta\left(T_{t_{k-1}}\right) \beta\left(T_{i_{k}}\right)^{2} \beta\left(T_{t_{k+1}}\right) \ldots \beta\left(T_{t_{p}}\right) \\
& =I_{p+1}\left(\chi_{\varepsilon}\right)+\sum_{k=1}^{p} \sum a_{i_{1} \ldots i_{p}} b_{i_{k}} \beta\left(T_{i_{i}}\right) \ldots \beta\left(T_{i_{k-1}}\right) m\left(T_{i_{k}}\right) \beta\left(T_{i_{k+1}}\right) \ldots \beta\left(T_{i_{p}}\right) \\
& +\sum_{k=1}^{p} \sum a_{i_{1} \ldots i_{p}} b_{i_{k}} \beta\left(T_{i_{1}}\right) \ldots \beta\left(T_{i_{k-1}}\right)\left(\beta\left(T_{i_{k}}\right)^{2}-m\left(T_{i_{k}}\right)\right) \beta\left(T_{i_{k+1}}\right) \ldots \beta\left(T_{i_{p}}\right) \\
& =I_{p+1}\left(\chi_{\varepsilon}\right)+\sum_{k=1}^{p} I_{p-1}(\varphi \underset{(k)}{\underset{x}{\times}} \phi)+\sum R_{k} \\
& \left\|I_{p+1}\left(\chi_{\varepsilon}\right)-I_{p+1}(\varphi \psi)\right\|^{2}=\underline{\mid p}\left\|\chi_{\varepsilon}-\varphi \cdot \psi\right\|^{2} \\
& =\sum \sum_{k=1}^{p} \sum a_{i_{1} \ldots i_{p}}^{2} b_{i_{k}}^{2} m\left(T_{i_{1}}\right) \ldots m\left(T_{i_{k-1}}\right) m\left(T_{i_{k}}\right)^{2} \ldots m\left(T_{i_{p}}\right) \\
& \leqq p A^{2} B^{2}\left(\sum m\left(T_{i}\right)\right)^{p-1} \cdot\left(\sum m\left(T_{i}\right)\right)^{2} \\
& \leqq \varepsilon p A^{2} B^{2}\left(\sum m\left(T_{i}\right)\right)^{p}=\varepsilon p A^{2} B^{2} m(S)^{p} \\
& \left\|R_{k}\right\|^{2}=c \sum a_{i_{1} \ldots i_{p}}^{2} b_{i_{k}}^{2} m\left(T_{i_{1}}\right) \ldots m\left(T_{i_{k-1}}\right) m\left(T_{i_{k}}\right)^{2} \ldots m\left(T_{i_{p}}\right) \\
& \left(c=\frac{1}{2 \pi} \int_{-\infty}^{\infty}\left(x^{2}-1\right)^{2} e^{-x^{2} / 2} d x\right), \varliminf^{\prime} \varepsilon c A^{2} B^{\circ} m(S)^{n} \text {. }
\end{aligned}
$$

Thus we obtain, as $\varepsilon \longrightarrow 0, \quad I_{p}(\varphi) \cdot I_{1}(\psi)=I_{p+1}(\varphi \psi)+\sum_{k=1}^{2} I_{p-1}(\varphi k \psi)$.

Let $\varphi$ and $\psi$ any functions respectively in $L^{2}\left(T^{p}\right)$ and $L^{2}(T)$. By virtue of Theorem 2.1 we can find special elementary functions $\varphi_{n} \in L^{2}\left(T^{p}\right)$ and $\phi_{n} \in L^{2}(T)$ such that $\quad\left\|\varphi_{n}-\varphi\right\| \rightarrow 0, \quad\left\|\psi_{n}-\psi\right\| \rightarrow 0$.

By the above argument we have

$$
I_{p+1}\left(\varphi_{n} \phi_{n}^{\prime}\right)=I_{p}\left(\varphi_{n}\right) I_{1}\left(\psi_{n}\right)-\sum_{k=1}^{p} I_{p-1}\left(\varphi_{n} \times \phi_{(k)}\right)
$$

By making use of (3.2), (3.3) and $\left(I .3^{\prime}\right)(\S 2)$ we obtain

$$
\begin{aligned}
& \left\|I_{p+1}\left(\varphi_{n} \psi_{n}\right)-I_{p+1}(\varphi \psi)\right\|_{1}=\left\|I_{p+1}\left(\varphi_{n} \psi_{n}-\varphi \psi\right)\right\|_{1}\left(\|\cdot\|_{1} \text { being the } L_{1} \text {-norm }\right) \\
& \leqq\left\|I_{p+1}\left(\varphi_{n} \psi_{n}-\varphi \psi\right)\right\| \leqq \sqrt{\underline{\underline{p+1}}\left\|\varphi_{n} \psi_{n}-\varphi \psi\right\|} \\
& \leqq \sqrt{\underline{p+1}}\left\|\varphi_{n}\left(\psi_{n}-\psi\right)\right\|+\sqrt{\underline{p+1}}\left\|\left(\varphi_{n}-\varphi\right) \psi\right\|
\end{aligned}
$$


Multiple Wiener integral

165

$$
\begin{aligned}
& =\sqrt{\underline{p+1}}\left\|\varphi_{n}\right\| \cdot\left\|\psi_{n}-\psi\right\|+\sqrt{\underline{p+1}}\left\|\varphi_{n}-\varphi\right\| \cdot\|\psi\| . \\
& \left\|I_{p}\left(\varphi_{n}\right) \cdot I_{1}\left(\psi_{n}\right)-I_{p}(\varphi) \cdot I_{1}(\psi)\right\|_{1} \leqq\left\|I_{p}\left(\varphi_{n}\right) I_{1}\left(\psi_{n}-\psi\right)\right\|_{1} \\
& +\left\|I_{p}\left(\varphi_{n}-\varphi\right) I_{1}(\psi)\right\|_{1} \\
& \leqq\left\|I_{p}\left(\varphi_{n}\right)\right\| \cdot\left\|I_{1}\left(\phi_{n}-\psi\right)\right\|+\left\|I_{p}\left(\varphi_{n}-\varphi\right)\right\| \cdot\left\|I_{1}(\phi)\right\| \\
& \leqq \sqrt{\underline{p} \|}\left\|\varphi_{n}\right\| \cdot\left\|\psi_{n}-\psi\right\|+\sqrt{\underline{p}}\left\|\varphi_{n}-\varphi\right\| \cdot\|\psi\| \text {. } \\
& \left\|I_{p-1}\left(\varphi_{n} \underset{(k)}{\times} \phi\right)-I_{p-1}(\underset{(k)}{\times} \psi)\right\|_{1} \leqq\left\|I_{p-1}\left(\varphi_{n} \underset{(k)}{\times} \psi_{n}-\varphi \underset{(k)}{\times} \psi\right)\right\| \\
& \leqq \sqrt{p-1}\left\|\varphi_{n} \underset{(k)}{\times} \psi_{n}-\varphi \underset{(k)}{\times} \phi\right\| \\
& \leqq \sqrt{\underline{p-1}}\left\|\left(\varphi_{n}-\varphi\right) \underset{(k)}{\times} \psi_{n}\right\|+\sqrt{\underline{p-1}}\left\|\varphi \underset{(k)}{\times}\left(\psi_{n}-\psi\right)\right\| \\
& \leqq \sqrt{\underline{p-1}}\left\|\varphi_{n}-\varphi\right\| \cdot\left\|\psi_{n}\right\|+\sqrt{\underline{1 p-1}}\|\varphi\| \cdot\left\|\psi_{n}-\phi\right\| .
\end{aligned}
$$

Thus we see that (3.4) is true in the general case by letting $n$ tend to $\infty$ in $(3.5)$.

Proof of Theorem 3.1. We make use of the mathematical induction with regard to $p_{1}+\ldots+p_{n}$. The theorem is trivially true in case $p_{1}+\ldots$ $+p_{n}=0$ or 1. It suffices to show that (3.1) is also true for $p_{1}+\cdots+p_{n}$ $=p+1$ under the assumption that (3.1) is valid for $p_{1}+\ldots+p_{n}=p-1, p$. We may suppose that $p_{1} \geqq 1$ with no loss of generality.

If we put, in Theorem 3.2,

$$
\begin{aligned}
\varphi\left(t_{1}, \ldots, t_{p}\right)= & \varphi_{1}\left(t_{1}\right) \ldots \varphi_{1}\left(t_{p_{1}-1}\right) \varphi_{2}\left(t_{p_{1}}\right) \ldots \varphi_{2}\left(t_{p_{1}+p_{2}-1}\right) \ldots \\
& \times \varphi_{n}\left(t_{p_{1}+\cdots+p_{n-1}}\right) \ldots \phi_{n}\left(t_{p_{1}}+\cdots \cdot p_{n}-1\right) \\
\psi(t)= & \varphi_{1}(t)
\end{aligned}
$$

we obtain, by the assumption of induction

$$
\begin{aligned}
& \int \ldots \int \varphi\left(t_{1}, \ldots, t_{p}\right) \varphi_{1}(t) d \beta\left(t_{1}\right) \ldots d \beta\left(t_{p}\right) d \beta(t) \\
& =\int \ldots \int \varphi\left(t_{1}, \ldots, t_{p}\right) d \beta\left(t_{1}\right) \ldots d \beta\left(t_{p}\right) \int \varphi_{1}(t) d \beta(t)
\end{aligned}
$$

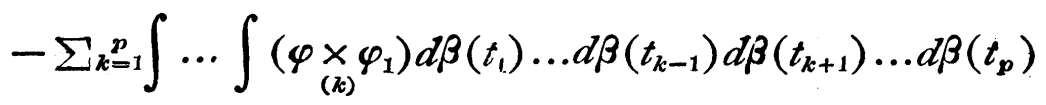




$$
\begin{aligned}
& =\prod_{\nu=2}^{n} \frac{H_{p_{\nu}}\left(\frac{1}{\sqrt{2}} \int \varphi_{\nu}(t) d \beta(t)\right)}{\sqrt{2^{p_{\nu}}}} \cdot \frac{H_{p_{1}-1}\left(\frac{1}{\sqrt{2}} \int \varphi_{1}(t) d \beta(t)\right)}{\sqrt{2^{p_{1}-1}}} \\
& \times \prod_{\nu=2}^{n} \frac{\int \varphi_{1}(t) d \beta(t)+\left(p_{1}-1\right)}{H_{p_{\nu}}\left(\frac{1}{\sqrt{2}} \int \varphi_{\nu}(t) d \beta(t)\right)} \cdot \frac{H_{p_{2}-2}\left(\frac{1}{\sqrt{2}} \int \varphi_{1}(t) d \beta(t)\right)}{\sqrt{2}^{p_{\nu}^{p_{\nu}}}} \\
& =\prod_{\nu=1}^{n} \frac{H_{p_{\nu}}\left(\frac{1}{\sqrt{2}} \int \varphi_{\nu}(t) d \beta(t)\right)}{\sqrt{2}^{p_{\nu}}}
\end{aligned}
$$

in considering

$$
\int \varphi\left(t_{1}, \ldots, t_{p}\right) \varphi_{1}\left(t_{k}\right) m\left(d t_{k}\right)=\left\{\begin{array}{cc}
\varphi_{1}\left(t_{1}\right) \ldots \varphi_{1}\left(t_{k-1}\right) \varphi_{1}\left(t_{k+1}\right) \ldots \varphi_{1}\left(t_{p_{1}-1}\right) \varphi_{2}\left(t_{p_{1}}\right) \\
\ldots \varphi_{n}\left(t_{p_{1}+\ldots .+p_{n}-1}\right) & \left(1 \leqq k \leqq p_{1}-1\right) \\
0 \quad\left(k \geqq p_{1}\right) &
\end{array}\right.
$$

and

(which follows from the orthonormality of $\left\{\varphi_{1}, \ldots, \varphi_{n}\right\}$ )

$$
H_{p_{1}}\left(\frac{x}{\sqrt{2}}\right)=\sqrt{2} H_{p_{1}}\left(\frac{x}{\sqrt{2}}\right)-2\left(p_{1}-1\right) H_{p_{1}-2}\left(\frac{x}{\sqrt{2}}\right)
$$

(which is the recursion formula of Hermite polynomials).

\section{§4. Orthogonal development of $L_{2}$-functionals of $\beta$}

by multiple Wiener integrals.

A mapping from $R^{B^{*}}$ into the complex number space $K$ is called $B$-measurable if the inverse image of any Borel set in $K$ is a $B$-measurable set in $R^{B *}$, which is a set belonging to the least complete additive class that contains all the Borel cylinder sets in $R^{B^{*}}$. A complex-valued random variable $\xi(\omega)$ is a $B$-measurable function of $\beta$ if it is expressible in the from :

$$
\hat{\boldsymbol{\xi}}(\omega)=f\left(\beta(E, \omega), E \in \boldsymbol{B}^{*}\right),
$$

for any $\omega, f$ being a $B$-meassurable mapping from $R^{B^{*}}$ into $K$. 
Furthermore if

$$
\|\xi\|^{2} \equiv \int|\xi(\omega)|^{2} d \omega<\infty
$$

then we say that $\xi(\omega)$ is an $L_{2}$-functional of $\beta$.

Theorem 4.1 (R. H. Cameron and W. T. Martin) ${ }^{8)}$ Let $\left\{\varphi_{\alpha}(t)\right\}$ be a complete orthonormal systein. Then any $L_{2}$-functional $\xi(\omega)$ of $\beta$ can be developed as follows:

$$
\xi=\sum_{p} \sum_{p_{1}+\cdots+p_{n}=p} \sum_{\alpha_{1}, \cdots, \alpha_{n}} a_{p_{1} \cdots p_{n}}^{\alpha \cdots \cdots \alpha_{n}} \prod_{\nu=1}^{n} H_{p_{\nu}}\left(\frac{1}{\sqrt{2}} \int \varphi_{\alpha_{\nu}}(t) d \beta(t)\right) .
$$

Cameron and Martin has shown this theorem in the case when $\boldsymbol{\beta}$ is a normal random measure derived from a brownian motion process, but their proof is available for our general case.

Theorem 4.2 Any $L_{2}$-functional $\xi$ of $\beta$ can be expressible in the form:

$$
\xi=\sum I_{p}\left(f_{p}\right)=\sum I_{p}\left(\tilde{f}_{p}\right),
$$

where $f$ is given by the following orthogonal development

$$
\begin{aligned}
f_{p}\left(t_{1}, \ldots, t_{p}\right)=\sqrt{2} \sum_{p_{1}+\ldots, p_{n}=p} \sum_{\alpha_{1}, \cdots, \alpha_{n}} a_{p_{1} \cdots p_{n}}^{\alpha \cdots \alpha_{n}} \varphi_{\alpha_{1}}\left(t_{1}\right) \ldots \varphi_{\alpha_{1}}\left(t_{p_{1}}\right) \\
\quad \times \varphi_{\alpha_{2}}\left(t_{p_{1}+1}\right) \ldots \varphi_{\alpha_{2}}\left(t_{p_{1}+p_{2}}\right) \ldots \varphi_{\alpha_{n}}\left(t_{p_{1}+\cdots+p_{n}+1}\right) \ldots \varphi_{\alpha_{n}}\left(t_{p_{1}}+\cdots+p_{n}\right),
\end{aligned}
$$

$\left\{\varphi_{\alpha}\right\}$ and. $\left\{a_{p_{1} \cdots p_{n}}^{\alpha \cdots \cdots n_{n}}\right\}$ being the same as those appearing in Theorem 4.1.

Since $I_{p}\left(f_{p}\right)$ (or $\left.I_{p}\left(\tilde{f}_{p}\right)\right), p=0,1,2, \ldots$, are orthogonal to each other, (4.3) may be considered as an orthogonal development,

We shall give another method of defining the symmetric functions $\{\tilde{S}\}$ which satisfy $\xi=\sum I_{p}\left(\tilde{S_{p}}\right)$. Put

$$
F\left(\tilde{h}_{p}\right)=\frac{1}{\underline{1 p}}\left(\xi, I_{p}\left(\tilde{h}_{p}\right)\right), \tilde{h} \in \tilde{L}^{2}\left(T^{p}\right),
$$

where $\tilde{L}_{\overline{\mathrm{c}}}\left(T^{p}\right)$ is the totality of symmetric functions in $L^{2}\left(T^{p}\right)$ which forms a closed linear subspace of $L^{2}\left(T^{p}\right)$.

8) R. H. Cameron and W. T. Martin: The orthogonal development of non-linear functionals in series of Fourier-Hermite functions. 
Then $F_{p}$ is a bounded linear functional on $\tilde{L^{2}}\left(T^{p}\right)$, since

$$
\begin{gathered}
F_{p}\left(a \tilde{h}_{p}+b \tilde{g}_{p}\right)=a F_{p}\left(\tilde{h}_{p}\right)+b F_{p}\left(\tilde{g}_{p}\right) \\
\left|F_{p}\left(\tilde{h}_{p}\right)\right| \leqq \frac{1}{\mid p}\|\xi\| \cdot\left\|I_{p}\left(\tilde{h}_{p}\right)\right\|=\|\xi\| \cdot\left\|\tilde{h}_{p}\right\| .
\end{gathered}
$$

By Riesz-Fischer's theorem in Hilbert space, we can find $\tilde{s}_{p} \in \tilde{L}^{2}\left(T^{p}\right)$ such that

$$
F_{p}\left(\tilde{h}_{p}\right)=\left(\tilde{s}_{p}, \tilde{h}_{p}\right)
$$

By $\frac{(4.3)}{\bullet}$ we have $F_{p}\left(\tilde{h}_{p}\right)=\frac{1}{\underline{p}}\left(I_{p}\left(\tilde{f}_{p}\right), I_{p}\left(\tilde{h}_{p}\right)\right)=\left(\tilde{f}_{p}, \tilde{h}_{p}\right)$.

Thus we have

$$
\left(\tilde{s}_{p}, \tilde{h}_{p}\right)=\left(\tilde{f_{p}}, \tilde{h}_{p}\right) \text { for } h_{p} \in \tilde{L}^{2}\left(T^{p}\right),
$$

which proves $\tilde{s}_{p}=\tilde{f}_{p}$.

From the above argument follows at once

Theorem 4.3. $\xi=\sum I_{p}\left(f_{p}\right)=\sum I_{p}\left(g_{p}\right)$ implies $\tilde{f}_{p}=\tilde{g}_{p}$.

\section{§. The case of a brownian motion process.}

Let $\beta(t), a<t<b$, be a brownian motion process.

If we put

$$
\beta(E)=\int c_{R}(t) d \beta(t),
$$

where $c_{B}(t)$ is the characteristic function of the set $E$ and the integral is the so-called Wiener integral. Then $\beta(E)$ is a normal random measure on $T=(a, b)$, the measure $m$ on $T$ being the so-called Lebesgue measure, which clearly fulfills the continuity-condition.

Let $f\left(t_{1}, \ldots, t_{p}\right) \in L^{2}\left(T^{p}\right)$. Then we can consider

$$
I=\int \ldots \int f\left(t_{1}, \ldots, t_{p}\right) d \beta\left(t_{1}\right) \ldots d \beta\left(t_{p}\right) .
$$

Theorem 5.1. The above multiple lliener integral $I$ is expressible in the form of iterated stochastic intgrals

9) loc. cit. 2). 


$$
\left.I=\underline{p} \int_{a}^{b}\left(\int_{a}^{t_{p}}\left(\ldots \int_{a}^{t_{3}}\left(\int_{a}^{t_{2}} f\left(t_{1}, \ldots, t_{p}\right) d \beta\left(t_{1}\right)\right) d \beta\left(t_{2}\right)\right) \ldots\right) d \beta\left(t_{p-1}\right)\right) d \beta\left(t_{p}\right)
$$

Proof. If $f$ is a special elementary function, this theorem is easily verified by the definitions. In the general case we can show it by approximating $f$ with a special elementary function and making use of the properties of multiple Wiener integrals and stochastic integral.

Any Wiener functional of the brownian motion process is an $L_{2^{-}}$ functional of the normal random measure derived from it, and vice versa. Therefore we see that Theorem 4.2 gives an orthogonal development of Wiener functionals.

I express my hearty thanks to Mr. H. Anzai for his friendly aid and valuable suggestions.

Mathematical Institute, Nagoya University. 